\title{
FEATURES OF FORMATION AND PROGRESSION OF CHRONIC KIDNEY DISEASE IN CHILDREN WITH PYELONEPHRITIS AND VESICOURETERAL REFLUX
}

\author{
Morozova $O$.
}

Kharkiv National Medical University, Kharkiv, Ukraine

\begin{abstract}
Vesicoureteral reflux (VUR) is observed in $40 \%$ of children with pyelonephritis and is one of the leading causes of its recurrent course, which subsequently leads to chronic kidney disease. The purpose of this study was to determine the peculiarities of the formation and progression of chronic kidney disease in children with pyelonephritis and vesicoureteral reflux. The clinical material from 141 children aged from 6 months to 17 years with grade I-V VUR in the period of clinical and laboratory remission of pyelonephritis was analyzed. The study showed that the risk of developing scarring of renal parenchyma in children with high-grade VUR was 8 times higher than in children with grade I-II VUR. And the risk of developing scarring of the renal parenchyma in patients with grade V VUR is 3.8 times higher than in children with grade III-IV VUR. In recurrent pyelonephritis, the risk of scarring of the renal parenchyma is 1.8 times higher than in one episode of inflammation. In patients with a high grade of reflux, the risk of recurrent pyelonephritis is 2.6 times higher than in children with grade I-II VUR. In patients with pyelonephritis and high-grade VUR, with signs of systemic undifferentiated connective tissue dysplasia, the risk of developing scarring of the renal parenchyma is 33.9 times higher. Depending on the grade of VUR and the presence of signs of scarring of the renal parenchyma, the degree of CKD increases, which reflects the functional state and severity of pathological changes in the kidneys.

Formation and progression of chronic kidney disease in children with pyelonephritis and VUR depends on the course of pyelonephritis, the grade of VUR and presence of signs of scarring in the renal parenchyma.
\end{abstract}

Keywords: Vesico-ureteral reflux, renal scarring, pyelonephritis, children.

Introduction. Vesicoureteral reflux (VUR) is observed in $40 \%$ of children with pyelonephritis and is one of the leading causes of its recurrent course [1, 2]. Recurrent course and chronic interstitial inflammation of the kidneys, especially secondary to VUR, initiates the development and progression of nephrosclerosis in 30-60 \% of patients, leading to chronic kidney disease, and subsequently chronic renal failure in $25-60 \%$ of patients [3-6]. Despite the fact that VUR has been studied for more than a century, no pathognomonic symptoms have yet been detected. It is for this reason that VUR is often diagnosed and treated at late stages, as late as in the presence of signs of scarring of the renal parenchyma. A lot of studies have been carried

Corresponding Author:

Morozova Oksana, MD,

Assistant of Department of Pediatrics

of Kharkiv National Medical Univeristy, Ukraine.

E-mail: oksanamorozova1612@gmail.com out over the last decade to find early diagnostic criteria for kidney damage in children with VUR $[7,8]$. However, there is a lack of information on the peculiarities of the formation and progression of chronic kidney disease in children with pyelonephritis and vesicoureteral reflux.

2. Purposes, subjects and methods:

2.1. Purpose - is to determine the peculiarities of the formation and progression of chronic kidney disease in children with pyelonephritis and vesicoureteral reflux.

2.2. Subjects \& Methods. The study involved assessment of clinical material from 141 children aged 6 months to 17 years with grade I-V VUR in the period of clinical and laboratory remission of pyelonephritis (more than 3 months after the last episode of exacerbation). Taking into account the grade of VUR and the method of its correction, the examined children were divided into three groups: Group 1 ( $n=24$ ) included patients with pyelonephritis and grade I-II VUR after or at the stage of conservative treatment, 
Group $2(\mathrm{n}=87)$ comprised children with pyelonephritis and grade III-IV VUR after endoscopic correction, Group $3(n=30)$ included children with pyelonephritis and grade V VUR after surgical correction of VUR. Statistical analysis was performed using Stat Soft STATISTICA Version 7 (Tulsa, OK). The planned clinical trial was approved by the Medical Ethics Committee of Kharkiv National Medical University and was conducted in accordance with the guidelines of Helsinki Declaration. All participants and/or their parents gave written informed consent to participate.

Conflict of interests. There is no conflict of interests.

3. Results and discussion. Assessment of age distribution showed that the number of children of junior and preschool age $(p<0.001)$ (Table) was statistically more significant, which corresponded to world statistics on the age distribution of patients with VUR [9-13]. literature data [17]. The researchers provide evidence that the incidence of chronic pyelonephritis and VUR is accompanied by a high genealogical index [18]. Family histories of the patients under examination showed the presence of congenital malformations of the urinary organs, pyelonephritis and urolithiasis in parents and / or close relatives of $55((39.01 \pm 9.95) \%)$ patients.

Physical examination of patients with pyelonephritis and VUR did not show any probable differences between accelerated or delayed physical development according to individual components. The study showed that 33 $((24.40 \pm 3.56) \%)$ patients of the main group had a tendency to allergic reactions, 82 ((58.16 \pm 4.15) \%) patients had chronic foci of infections of ENT organs, $47(33.33 \pm 3.97) \%$ in the oral cavity, which can significantly affect the protective forces of the body and contribute to the subsequent relapse of pyelonephritis. In 110 $((78.01 \pm 8.45) \%)$ patients, pyelonephritis

Distribution of patients with pyelonephritis and VUR by age and gender

\begin{tabular}{|l|c|c|c|c|c|c|c|c|}
\hline \multirow{2}{*}{ Gender } & \multicolumn{2}{|c|}{6 months -6 years } & \multicolumn{2}{|c|}{$7-11$ years } & \multicolumn{2}{|c|}{$12-17$ years } & \multicolumn{2}{c|}{ Total } \\
\cline { 2 - 9 } & $\mathrm{n}$ & $\mathrm{p} \% \pm \mathrm{s}_{\mathrm{p} \%}$ & $\mathrm{n}$ & $\mathrm{p} \% \pm \mathrm{s}_{\mathrm{p} \%}$ & $\mathrm{~N}$ & $\mathrm{p} \% \pm \mathrm{s}_{\mathrm{p} \%}$ & $\mathrm{~N}$ & $\mathrm{p} \% \pm \mathrm{s}_{\mathrm{p} \%}$ \\
\hline Boys & 19 & $13.5 \pm 2.9$ & 14 & $9.9 \pm 2.5$ & 5 & $3.6 \pm 1.6$ & 38 & $26.9 \pm 3.7$ \\
\hline Girls & 65 & $46.1 \pm 4.2$ & 22 & $15.6 \pm 4.3$ & 16 & $11.4 \pm 2.6$ & 103 & $73.1 \pm 3.7$ \\
\hline Total & 84 & $59.7 \pm 4.1^{*}$ & 36 & $25.5 \pm 3.6^{*}$ & 21 & $14.9 \pm 2.9^{*}$ & 141 & $100.0 \pm 0.0$ \\
\hline
\end{tabular}

Notes: $\mathrm{n}$ is the number of observations; $\mathrm{p} \%$ is sample share in percent; $\mathrm{sp} \%$ is statistical error of the sample share in percent; * is percent from the total number of patients.

This may be a reflection of the fact that the debut of pyelonephritis secondary to VUR most often occurs at an early age. Gender distribution analysis showed that, regardless of age, in all groups of children with pyelonephritis and VUR, the number of girls was statistically more significant than boys $(\mathrm{p}<0.001)$, which does not contradict the data of the European Association of Urologists (EAU, 2012) [7].

According to the European Registry, provided by the EDTA (European Dialysis and Transplant Association), the incidence of congenital and hereditary kidney diseases has increased in recent decades, contributing to the development of CKD. The progressive increase in the number of patients with VUR is attributed to the pathology of antenatal development [14-16]. Chronic intrauterine fetal hypoxia has been found to be a risk factor for the development of renal dysembryogenesis. Assessment of medical records concerning the course of pregnancy and labor, heredity and premorbid background testify to the negative influence of adverse factors on the formation of congenital malformations of urinary organs, which does not contradict the manifested at a younger age $(\mathrm{p}<0.0001)$. Patients of all groups with the same frequency came for examination with changes in clinical analysis of urine in the form of leukocyturia and fever of unknown origin. Hyperthermia as the first manifestation of pyelonephritis was observed predominantly in young children, in rare cases, fever was combined with dysuric signs, but no significant intergroup differences were observed. Pain syndrome and dysuric signs in manifestation of pyelonephritis were predominantly observed in adolescents. In one child, the reason for nephrourological examination, in addition to hyperthermia, was an urgent surgical intervention due to carbuncle of the left kidney.

According to the results of voiding cystography, higher grades of VUR (III, IV, V) $(p<0.0001)$ were observed more frequently, without a statistically significant difference between the lesion side or bilateral localization (total $\mathrm{p}<0.05$ ), but unilateral lesion was more frewent than bilateral in all grades of VUR $(\mathrm{p}=0.0001)$. In 39 (27.66 $\pm 3.77 \%)$ of the subjects with pyelonephritis and VUR, there were signs of scarring of the renal parenchyma. The risk of 
developing scarring of the renal parenchyma in children with high grades of VUR (III, IV, V) is 8 times higher than in children with grade III VUR (RR = 8.00 [1.16; 55.44]). And the risk of developing scarring of the renal parenchyma in patients with grade V VUR is 3.8 times higher than in children with grade III-IV $(\mathrm{RR}=3.38$ [2.11; 5.43], $\mathrm{p}<0.05$ ), which does not contradict the literature [19, 20].

In the vast majority of subjects $(68.79 \pm 3.90 \%$, $\mathrm{p}<0.0001$ ), pyelonephritis secondary to VUR had a recurring course with the number of episodes from 2 to 6 (Figure). In recurring course of with grade V VUR without a significant difference between patients with signs of scarring of the renal parenchyma and without them $(p=0.1063, p=0.77345$, respectively). Other congenital renal abnormalities, such as doubling of pyelocalyceal system, Fraley syndrome, were diagnosed in all groups of the children under examination without intergroup differences.

In the vast majority of patients, 76 (53.90 \pm $4.26 \%$ ) had signs of connective tissue dysplasia, which, according to literature data, contributes to the recurrence of pyelonephritis [22]. Statistically more often $(p<001)$ signs of undifferentiated

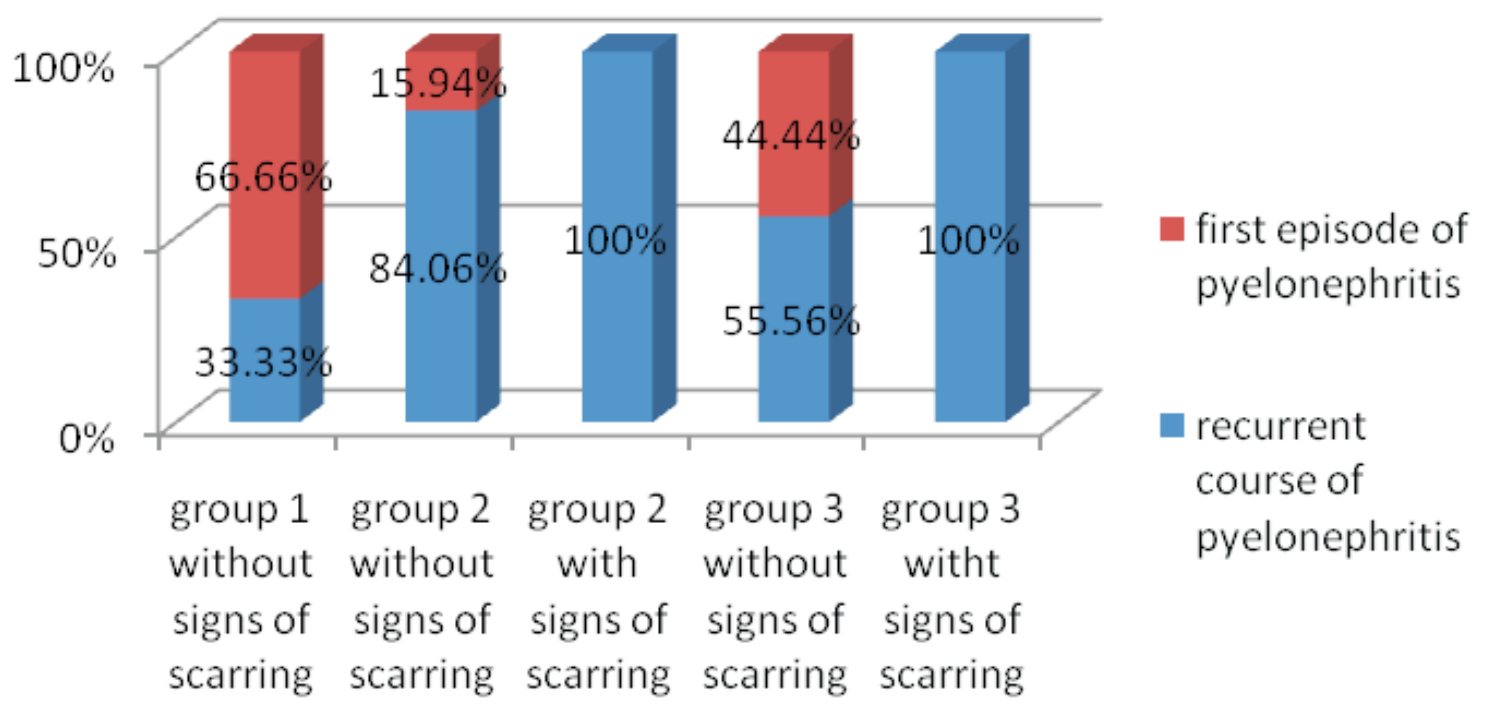

The ratio of patients with different grades of VUR depending on the course of pyelonephritis

pyelonephritis, the risk of scarring of the renal parenchyma is 1.8 times $(\mathrm{RR}=1.81[1,50 ; 2,18])$ higher than in one episode of inflammation. The risk of recurrent pyelonephritis in children with a high reflux rate is 2.6 times $(\mathrm{RR}=2.55$ [1.35; 4.80]) higher than in children with grade I-II VUR.

Besides, $54.61 \pm 10.16 \%$ patients were found to have concomitant kidney disease secondary to VUR. Namely: 68 (48.23 $\pm 10.19 \%)$ children had dysmetabolic nephropathy, which was caused not only by metabolic disorders, but also by the presence of VUR, which created an obstacle to the outflow of urine. Carbuncle of the kidneys, as a purulent-inflammatory disease, was observed in 1 child with a bilateral grade III VUR. Urolithiasis was observed in isolated cases, but in children with grade III-V VUR and mainly in patients with signs of renal parenchymal scarring, which may be due to reflux nephropathy, as also confirmed by other studies [21]. Refluxing megaureter and hydronephrosis were predominantly $(\mathrm{p}<0.0001)$ observed in patients connective tissue dysplasia were observed in patients with signs of scarring of kidney parenchyma, which scientists are paying attention to. The calculations showed that in children with pyelonephritis and high grade of VUR with signs of systemic undifferentiated dysplasia of the connective tissue, the risk of developing scarring of the renal parenchyma was 33.9 times higher ( $R R=33.87$ [4.78; 239.82]). The study detected a tendency to an increase in the percentage of children with recurrent pyelonephritis, depending on the grade of VUR and the presence of signs of scarring of the renal parenchyma.

It is noteworthy that, depending on the grade of VUR and the presence of signs of scarring of the kidney parenchyma, the number of patients with a deflection of GFR from the normative indexes increases. According to the results of the analysis of indices of the state of glomerular filtration and tubular renal function, it was found that in the subjects of Group 1, renal function was not disturbed; Group 2 and 3 had a significant 
number of patients with a deflection of the GFR from normative indices and with a decrease in concentration kidney function, with the highest specific gravity among patients with signs of renal parenchyma scarring. Thus, depending on the grade of VUR and the presence of signs of scarring of the kidney parenchyma in the affected patients, the degree of CKD increases, which reflects the functional state and severity of pathological changes in the kidneys.

Conclusions. Pathological course of pregnancy, aggravated heredity on the part of kidney diseases in parents and close relatives, high grades of VUR, recurrent pyelonephritis and signs of systemic undifferentiated connective tissue dysplasia were identified as risk factors for the formation and progression of chronic kidney disease in children with pyelonephritis and vesicoureteral reflux. This gives an opportunity to identify them as a risk group for nephrosclerosis, which is the cause of progression of chronic kidney disease towards the terminal stage of chronic renal failure. Thus, the formation and progression of chronic kidney disease in children with pyelonephritis and VUR depend on the course of pyelonephritis, the degree of VUR and the presence of signs of scarring of the renal parenchyma.

\section{References:}

1. Salvatore Arena. Roberta Iacona, Pietro Impellizzeri, Tiziana Russo, Lucia Marseglia, Eloisa Gitto, Carmelo Romeo (2016). Physiopathology of vesico-ureteral reflux. Italian Journal of Pediatrics. 42:103. doi:10.1186/s13052-016-0316-X.

2. Samantha E. Bowen, Christine L. Watt, Inga J. Murawski, Indra R. Gupta, Soman N. Abraham. (2013). Interplay between vesicoureteric reflux and kidney infection in the development of reflux nephropathy in mice. Dis Model Mech. 6(4): 934-941. doi:10.1242/dmm.011650

3.Carpenter MA, Hoberman A, Mattoo TK. (2013). The RIVUR trial: profile and baseline clinical associations of children with vesicoureteral reflux. Pediatrics. 132(1):34-45. doi:10.1542/peds.20122301.

4. Becknell B., Schober M, Korbel L., Spencer J.D. The diagnosis, evaluation and treatment of acute and recurrent pediatric urinary tract infections. Expert Rev Anti Infect Ther.1(13):81-90. doi: 10.1586/ 14787210.2015.986097.

5. Paintsil E. (2013). Update on recent guidelines for the management of urinary tract infections in children: the shifting paradigm. Curr Opin Pediatr. 25(1):88-94. doi: 10.1097/MOP.0b013e32835c14cc.

6. Diamond D.A., Mattoo T.K. (2012). Endoscopic Treatment of Primary Vesicoureteral Reflux. N Engl J Med. 367:88-89. doi: 10.1056/NEJMc1204964.

7. Simoes e Silva A.C., Pereira À., Teixeira Ì ., Teixeira À. (2014). Chemokines as potential markers in pediatric renal diseases. Biomarkers in Kidney Disease. 1:1-9. doi: 10.1155/2014/278715.

8. Yŷmaz S., Ozçakar ZB., Kurt Dukur ED., Bulum B., Kavaz A., Elhan AH., Yalçýkaya F. (2016). Vesicoureteral reflux and renal scarring risk in children after the first febrile urinary tract infection. Nephron. 132(3):175-180. doi: 10.1159/000443536.

9. Xin Zhang, Hong Xu, Lijun Zhou, Qi Cao, Qian Shen, Li Sun, Xiaoyan Fang, Wei Guo, Yihui Zhai, Jia Rao, Mier Pa, Ruifang Zhao, Yunli Bi. (2014). Accuracy of Early DMSA Scan for VUR in Young Children With Febrile UTI. Pediatrics. 133:30-38. doi: 10.1542/peds.2012-2650

10. Michael Garcia-Roig, Derrick E. Ridley, Courtney McCracken, Angela M. Arlen, Christopher S. Cooper, Andrew J. Kirsch. (2017).Vesicoureteral reflux index: predicting primary vesicoureteral reflux resolution in children diagnosed after age 24 months. The Journal of Urology. 197(4):1150-1157.

doi: https://doi.org/10.1016/j.juro.

11. Anne-Sophie Blais, Stéphane Bolduc, Katherine Moore. (2017). Vesicoureteral reflux: From prophylaxis to surgery review. Urol Assoc J. 11:13-8. doi: http://dx.doi.org/10.5489/cuaj.4342

12. Keren R., Shaikh N., Pohl H., Gravens-Mueller L., Ivanova A., Zaoutis L., Hoberman A. (2015). Risk factors for recurrent urinary tract infection and renal scarring. Pediatrics. 136(1):13-21. doi: 10.1542/ peds.2015-0409

13. Fahimeh Ehsanipour, Minoo Gharouni, Ali Hoseinpoor Rafati, Maryam Ardalan, Neda Bodaghi, Hasan Otoukesh. (2012). Risk factors of renal scars in children with acute pyelonephritis. Braz J Infect Dis. 16(1):15-18. doi.org/10.1590/S1413-86702012000100003.

14. Guideline on pediatric urology. (2012). http://www.uroweb.org/ guidelines/online-guidelines/.

15. Hindryckx C., L. De Catte. (2016). Prenatal diagnosis of congenital renal and urinary tract malformations. Facts Views Vis Obgyn. 3(3):165-174. 
16. Ninoa F., Ilaria M., Noviello C., Santoro L., R?tsch I.M., Martino A., Cobellis G. (2013). Genetics of Vesicoureteral Reflux. Curr Genomics. 17(1):70-79. doi:10.2174/1389202916666151014223507.

17. Hila Milo Rasouly, Weining Lu. (2013). Lower urinary tract development and disease. Wiley Interdiscip Rev Syst Biol Med. 5(3):307-342.

doi:10.1002/wsbm.1212.

18. Groen In 't Woud S., Renkema KY., Schreuder MF., Wijers CH., van der Zanden LF., Knoers NV., Feitz WF., Bongers EM., Roeleveld N., van Rooij IA. (2016). Maternal risk factors involved in specific congenital anomalies of the kidney and urinary tract: A case-control study. Birth Defects Res A Clin Mol Teratol. 106(7):596-603. doi: 10.1002/bdra.23500.

19. Tain YL., Luh H., Lin CY., Hsu CN. (2016). Incidence and Risks of Congenital Anomalies of Kidney and Urinary Tract in Newborns: A Population-Based Case-Control Study in Taiwan. Medicine (Baltimore). 95(5):2659. doi: 10.1097/MD.0000000000002659.

20. Mei-Ju Chen, Hong-Lin Cheng, Yuan-Yow Chiou. (2013). Risk Factors for Renal Scarring and Deterioration of Renal Function in Primary Vesico-Ureteral Reflux Children: A Long-Term Follow-Up Retrospective Cohort Study. 8(2): e57954. doi: 10.1371/journal.pone.0057954.

21. Nader Shaikh, Jonathan C. Craig, Maroeska M. Rovers, (2014). Identification of Children and Adolescents at Risk for Renal Scarring After a First Urinary Tract InfectionA Meta-analysis With Individual Patient Data. JAMA Pediatr. 168(10):893-900. doi:10.1001/jamapediatrics.2014.637.

22. Louise K Isling, Bent Aalbæk, Malene Schrî der, Pàll S Leifsson. (2010). Pyelonephritis in slaughter pigs and sows: Morphological characterization and aspects of pathogenesis and aetiology. Acta Vet Scand. 52(1): 48. doi:10.1186/1751-0147-52-48.

23. Abbas Madani, Nooshin Kermani, Neamatollah Ataei, Seyed Taher Esfahani, Niloufar Hajizadeh, Zahra Khazaeipour, Sima Rafiei. (2012). Urinary calcium and uric acid excretion in childrenwith vesicoureteral reflux. Pediatr Nephrol. 27:95-99. doi 10.1007/s00467-011-1936-4.

Received: 20-Jun. - 2018

Accepted: 19-Sep. - 2018 